doi: $10.4248 / \operatorname{IJOS} 10013$

\title{
Role of the Chinese Herbal Medicine Xianhuayin on the Reversal of Premalignant Mucosal Lesions in the Golden Hamster Buccal Pouch
}

\author{
Yan-zhi Xu*, Yong-le Qiu, Zhi-guang An, Feng-ying Yang \\ Department of Stomatology, The Fourth Hospital of Hebei Medical University, Shijiazhuang, China
}

\begin{abstract}
Aim To investigate the role of the Chinese herbal medicine Xianhuayin on the reversal of 7,12-dimethylbenz[a]anthracene (DMBA)-induced premalignant mucosal lesions in the oral buccal pouch of golden hamsters.

Methodology The animals were randomly divided into a non-diseased control group $(n=5)$ and an experimental group including 50 animals in which the buccal mucosa had been painted with DMBA $(0.5 \%$ in acetone) to generate an oral mucosa premalignant lesion. Animals in the experimental group were further divided into Xianhuayin-treated group $(n=30)$, untreated premalignant lesion group $(n=10)$ and normal saline (NS)-treated group $(n=10)$. The cheek (buccal) pouch mucosa of the golden hamsters in each group was observed with light and electron microscopy eight weeks after intragastric administration with NS or Xianhuayin. Results In the non-diseased control group, the buccal mucosa was keratinized and stratified squamous epithelium under a light microscope. In the untreated premalignant lesion group, variable degrees of epithelial dysplasia was observed. The irregular epithelial mucosa gradually became distinct in the Xianhuayin-treated group. Scanning electronic
\end{abstract}

microscopic (SEM) analysis showed that surface of the cells exhibited honeycomb structures in the hamster of untreatedgroup. The cells were morphologically irregular, overlapped and loosened in the untreated premalignant lesion group. Most of the cell surface exhibited honeycomb structure in the Xianhuayin-treated group. Transmission electronic microscopic (TEM) analysis showed that buccal mucosal epithelial cells were morphologically regular in the non-diseased control group. Desmosomes and tonofibrils were reduced and the nucleus was morphologically irregular in the untreated premalignant lesion group. In the Xianhuayin-treated group, the widening intercellular gap was gradually reduced, desmosomes and the cells becoming morphologically regular. No significant difference was observed between the hamsters in NS-treated group and those in the untreated premalignant lesion group. Significant therapeutic efficacy was observed in the group receiving Xianhuayin.

Conclusion Xianhuayin is effective in the reversal of DMBA-induced premalignant lesions in the buccal pouch of golden hamsters.

Keywords Xianhuayin, oral premalignant lesion, golden hamster, buccal pouch mucosa

Received Sep. 15, 2009; Revision accepted Nov. 12, 2009

\section{Introduction}

Oral mucosal premalignant lesions are chronic focal lesions in the mouth, which include longterm ulceration, leukoplakia and erythema etc. Although these chronic lesions are not carcinomatous, they can progress to cancer under conditions of unfavorable stimulation and lack of immediate treatment.
In order to reduce the incidence of oral carcinoma, early diagnosis and prompt treatment of oral premalignant lesions is necessary. Although multiple therapies (e.g. drug, physical and surgical therapies) for oral premalignant lesions are currently available, they are not specific. Drug therapy is still the most favorable and extensively investigated therapy. In this study, we developed a buccal mucosal pre- 
malignant lesion model in the golden hamsters and subsequently treated these hamsters with the Chinese herbal medicine Xianhuayin. We investigated the role of Xianhuayin in the reversal of oral buccal mucosal premalignant lesions. We hypothesized that the application of Xianhuayin is effective for the treatment of oral mucosal premalignant lesions.

\section{Materials and Methods}

\section{Experimental animals}

A total of 55 SPF LVG Syrian golden hamsters (6 weeks old, weight: $80 \mathrm{~g}$ ) were provided by Beijing Vital River Experimental Animal Technology Co., Ltd (Beijing, China). Hamsters were raised in cages ( 5 hamsters/cage) at a temperature of $18-22^{\circ} \mathrm{C}$, humidity of $40 \%-60 \%$, sufficient light, and provision of refined feeds. Padding materials were changed 2-3 times per week. The requirements for the animals include a plump shape, normal development, thick and glossy clothing hair that clings to the body, bright eyes, activeness, physical agility and rapid response. The procedures for handling animals were in accordance with Guidance suggestion of caring laboratory animals enacted by the Ministry of Science and Technology of China (The Ministry of Science and Technology of the People's Republic of China, 2006).

\section{Grouping of the animals}

Animals were randomly divided into a nondiseased control group $(n=5)$ and an experimental group $(n=50)$. Animals in the experimental group were used to generate the oral mucosal premalignant lesion models. Of these 50 animals with oral mucosal premalignant lesions, 10 hamsters were treated with normal saline (NS-treated group); 10 hamsters were untreated (premalignant lesion group); 30 hamsters were treated with Xianhuayin (Xianhuayin-treated group).

\section{Generation of animal model with oral mucosal premalignant lesion}

The buccal mucosa of 50 hamsters in the experimental group was painted with 7,12-dimethylbenz[a]anthracene (DMBA, $0.5 \%$ in acetone) as described previously (Xie and Zhang, 1989). Briefly, the animals were fasted for food and water for 2 hours after DMBA was applied. Application with DMBA was performed three times a week for 6 weeks. The elasticity of buccal mucosa was reduced and the surface of the mucosa exhibited anabrotic, congested and streak-like ruffle after DMBA application. Histological analysis was performed by randomly collecting lesion tissues, identified through their visual manifestations. Oral premalignant lesion was confirmed according to the WHO standard (Zhou and Zhang, 1997).

\section{Preparation of Chinese herbal medicine Xian- huayin decoction}

Xianhuayin (Xu et al., 2009) was made of phellodendron amurense, villous amomum fruit, poria cocos, safflower and glycyrrhiza etc. based on a certain percentage of each component. Before decoction, all these components were immersed in 8-times volume of distilled water for 20 minutes. After heating and boiling, they were decocted with mild heating for 30 minutes (first decoction). The herb liquid was obtained by filtration and the filter residues were added with 6-times volume of water. The second decoction was performed for 20 minutes and the herb liquid was obtained by filtration. The herb liquid obtained from first and second decoctions were combined and concentrated until $1 \mathrm{~g} \cdot \mathrm{mL}^{-1}$ of crude drug was achieved. The concentrated herb liquid was stored at $4^{\circ} \mathrm{C}$ for future use.

\section{Procedures for treatment}

One week after DMBA treatment was stopped, Xianhuayin was intragastrically administered. Intragastic administration was performed once a day for 8 weeks. The animals in the NS group were treated with NS in a similar way. All the animals were sacrificed and the tissue samples were collected for histological analysis.

\section{Intragastic administration method}

We used a $1-5 \mathrm{~mL}$ syringe and metal blunt pinhead. The animal was fixed left hand. Skin of back and tail was then fixed on the left hand, to place the head and trunk vertical. The syringe was held in the right hand and from the oral cavity, gullet posterior wall, the syringe was inserted into the fistula cibalis, and then into the stomach. The 
needling length was $3.5-5.5 \mathrm{~cm}$ generally. The drug dosages given were based on the known value of human and animal, which were $1.7 \mathrm{~mL} \cdot \mathrm{kg}^{-1} \cdot \mathrm{d}^{-1}$ and $11.4 \mathrm{~mL} \cdot \mathrm{kg}^{-1} \cdot \mathrm{d}^{-1}$ respectively, according to human and animal body surface relative area formula conversions.

\section{Sample collection from animals}

The animals in each group were raised in usual fashion. Samples of hamsters from each group were collected at the same time after immediate injection with $1 \%$ of pentobarbital sodium solution. Samples (size: $1.0 \mathrm{~cm} \times 1.5 \mathrm{~cm} \times 0.1 \mathrm{~cm}$ ) of lesion and neighboring normal tissues were collected from anterolateral sites of both sides of the cheek pouch. Formalin-fixed samples were used for light microscopic analysis and glutaraldehyde-fixed samples were used for electronic microscopic analysis.

\section{Results}

\section{Premalignant lesion animal model generation}

Six weeks after treatment with DMBA acetone solution, in the experimental group, both sides of the cheek pouch mucosae of hamsters became adhered, lackin elasticity, congested, with anabrotic and streak-like ruffle. Histological analysis was performed by randomly collecting lesion tissues, identified through visual manifestations. Variable degree of epithelial dysplasia was observed under light microscopic .

\section{Xianhuayin therapeutic efficacy}

\section{Light microscopic observations}

Buccal mucosa of the normal hamsters in the non-diseased control group was keratinized and stratified squamous epithelium under light microscope. Lamellar connective tissues, and small amount of sporadic fibrocytes and blood vessels were present between epithelium and muscular layer (Figure 1). Variable degrees of epithelial dysplasia and simple hyperplasia were observed in the untreated premalignant lesion group eight weeks after painting with DMBA was stopped. Simple hyperplasia was featured in the increased layers of epithelium especially for the prickle and basal cells. Dysplasia was featured in the atypical change, disorganized arrangement of basal cells, enlargement and irregularity of the nucleus, with increased chromatins and the involvement in the inferior $1 / 2$ or 2/3 of epithelial layers (Xu et al., 2001) (Figure 2). Eight weeks after treatment with Xianhuayin, the irregular epithelial mucosa gradually became distinct, the layer of epithelium was recovered to normality, basilar membrane cells were regularly organized and the number of atypical cells was reduced (Figure 3). No significant changes were observed after hamsters were intragastrically treated with NS.

In the Xianhuayin-treated group, before treatment, variable degree of epithelial dysplasia was observed under light microscopy. Then, after treatment, simple hyperplasia was mainly observed in a high proportion, a difference with statistical significance $(P<0.05)($ Table 1$)$.

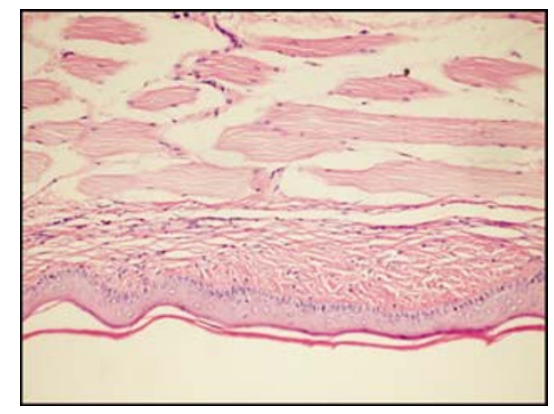

Figure 1 Light microscopic observation for the cheek pouch mucosa of hamsters in the untreated group

Mucosal epithelium was composed of 4-6 layers and the basal membrane was regularly arranged. $(\mathrm{HE} \times 200)$

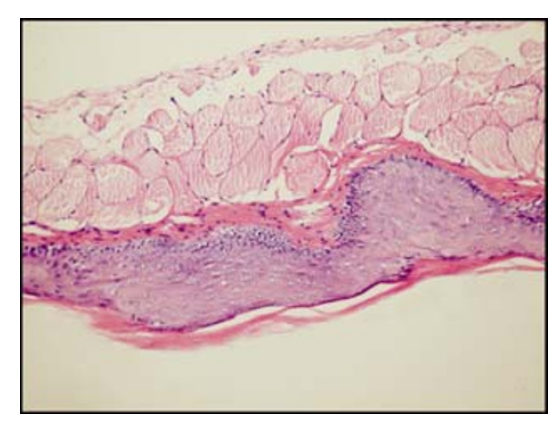

Figure 2 Light microscopic observation for the cheek pouch mucosa of hamsters in the untreated premalignant lesion group

The mucosal epithelium was thickened, basal cells were irregularly arranged, atypical cells appeared, the number of prickle cells increased and dropwise epithelial tag was observed. (HE $\times 200)$ 


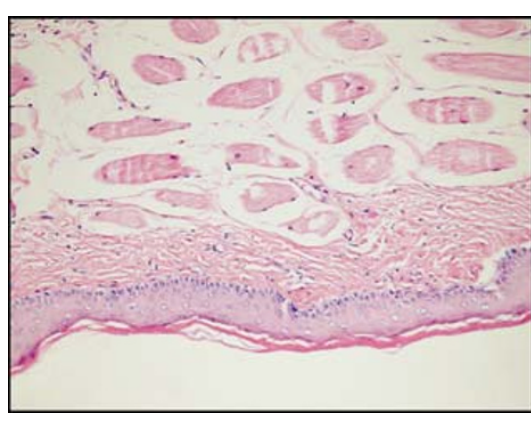

Figure 3 Light microscopic observation for the cheek pouch mucosa of hamsters in the Xianhuayintreated group

The thickness of the epithelial layers was uneven, basal cells were relatively regularly arranged, the number of atypical cells was reduced and nucleolus staining was gradually recovered to normal. $(\mathrm{HE} \times 200)$

Table 1 The comparison between before treatment and after treatment in the Xianhuayin-treated group

\begin{tabular}{lccc}
\hline $\begin{array}{c}\text { Xianhuayin-treated } \\
\text { group }\end{array}$ & $n$ & $\begin{array}{c}\text { Simple } \\
\text { hyperplasia }\end{array}$ & $\begin{array}{c}\text { Epithelial } \\
\text { dysplasia }\end{array}$ \\
\hline Before treatment & 30 & 2 & 28 \\
After treatment & 30 & 25 & 5 \\
\hline
\end{tabular}

$X^{2}=35.6229, \quad P<0.05$.

\section{SEM analysis}

Scanning electronic microscopic (SEM) analysis showed that in the non-diseased control group, the buccal mucosa contained polygonal cells and clear cell bridge. The surface of the individual cells exhibited honeycomb structures (Figure 4). Eight weeks after painting with DMBA was stopped, the epithelial cells of hamsters in the untreated premalignant lesion group were morphologically irregular, overlapped and loosened. Swelling and widening micro-crests were irregularly arranged and appeared as gravel shape. The honeycomb structures disappeared (Figure 5). Eight weeks after intragastric administration with Xianhuayin, most of the cell surface exhibited honeycomb structures and continuous cellular border was observed in the Xianhuayin-treated group (Figure 6). No significant changes were observed over time in the NS-treated group compared to those in the untreated premalignant lesion group.

\section{TEM analysis}

Transmission electronic microscopic (TEM)

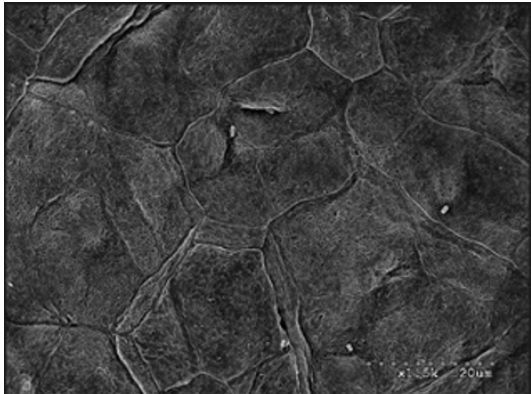

Figure 4 SEM analysis for the cheek pouch mucosa of hamsters in the untreated group

The shape of the mucosal cells was regular, cellular bridge was evident, cellular border was distinct and the surface of the cells exhibited a honeycomb structure. $($ SEM $\times 1500)$

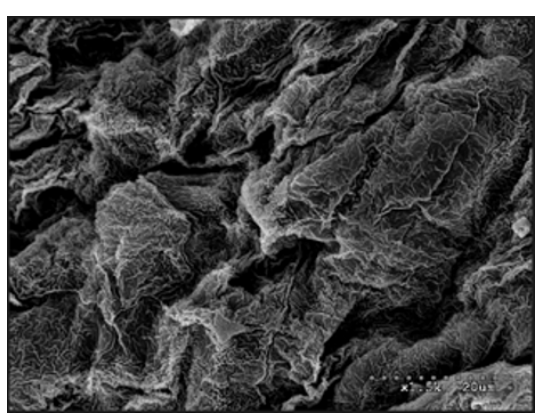

Figure 5 SEM analysis for the cheek pouch mucosa of hamsters in the untreated premalignant lesion group The shape of the mucosal cells was irregular, epithelial cells were loosened, micro-crests were swelling and widening and the honeycomb structure disappeared. $($ SEM $\times 1500)$

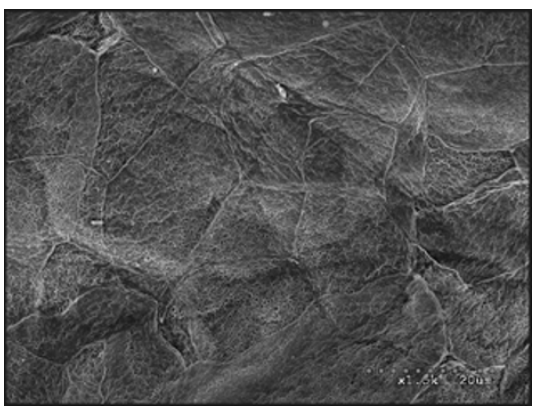

Figure 6 SEM analysis for the cheek pouch mucosa of hamsters in the Xianhuayin-treated group

The shape of the mucosal cells became clear, most of the cell surface exhibited honeycomb structures and continuous cellular border was observed. (SEM $\times 1500)$

analysis showed that in the non-diseased control group, buccal mucosal epithelial cells were morphologically regular, closely connected and contained abundant desmosomes. The tonofibrils were developed normally (Figure 7). In the untreated premalignant lesion group, desmosomes and tono- 
fibrils were significantly reduced, intercellular gap was increased, the shape of epithelial cells was irregular, the morphologically irregular nucleolus was marginally gathered and the caryotheca was invaginated (Figure 8). In the Xianhuayin-treated group, the widening intercellular gap became much reduced, desmosomes and tonofibrils were

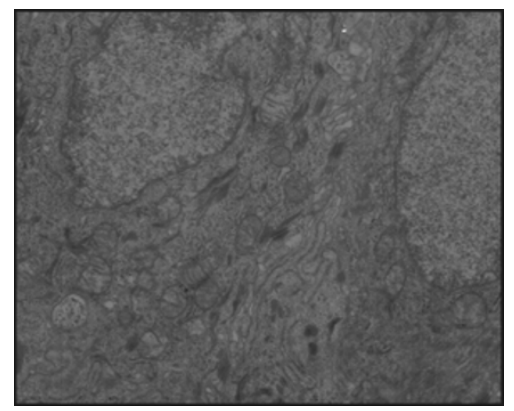

Figure 7 TEM analysis for the cheek pouch mucosa of hamsters in the untreated group

The mucosal epithelial cells were closely connected and the desmosomes and tonofibrils were abundant. (TEM $\times 10000$ )

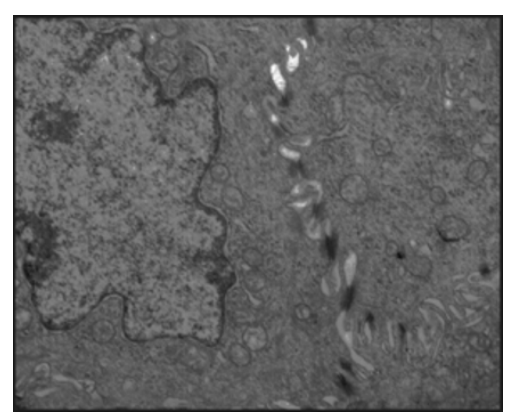

Figure 8 TEM analysis for the cheek pouch mucosa of hamsters in the untreated premalignant lesion group The intercellular gap between mucosal epithelial cells was widening, the shape of these cells was irregular and the number of desmosomes and tonofibrils was largely reduced. $($ TEM $\times 10000)$

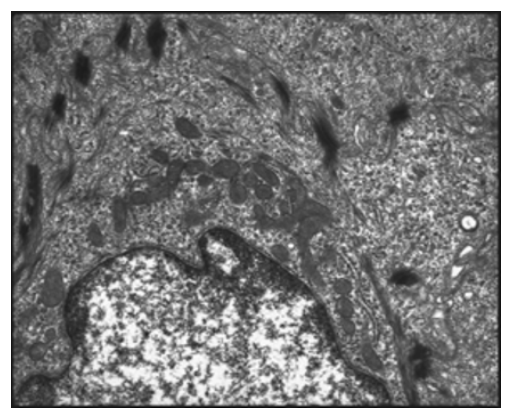

Figure 9 TEM analysis for the cheek pouch mucosa of hamsters in the Xianhuayin-treated group

The mucosal epithelial cells were morphologically becoming regular and the desmosomes and tonofibrils were abundant. $($ TEM $\times 10000)$ gradually becoming abundant and the cells were becoming morphologically regular (Figure 9). No significant difference was observed between the NS-treated group and those in the untreated premalignant lesion group.

\section{Discussion}

Oral premalignant lesions are those morphologically changed tissues with much higher incidence of carcinogenesis than corresponding normal tissues (Buajeeb et al., 2009). Based on the WHO classification, there are two main types of oral premalignant lesions: oral leukoplakia and oral erythroplakia. Oral premalignant lesions also include smoking-associated keratosis. Precancerous conditions, e.g. lichen planus, are also considered to be associated with premalignant lesions (Gao, 2008). Although there are multiple approaches to treat oral premalignant lesions, specific therapeutics are not available yet. No significant breakthroughs have been made with regard to the treatment of oral premalignant lesions by western medicines. Symptom relief can be achieved by local or systemic uses of cortical hormones. Western medicine also uses retinoic acid and other immunomodulators to treat oral premalignant lesions. However, these drugs are not curative. In addition, these drugs have severe side effects and the therapeutic efficiency is not stable. In addition, once the patients stops using these drugs, there is a recurrence of the oral premalignant lesions. Integrated traditional Chinese and western medicine is based on the uses of Chinese herbal medicine combined with hormone and immunomodulators. Recently, studies have been performed to investigate the application of Chinese herbal medicine for the treatment of oral premalignant lesions and a certain therapeutic efficacy has been achieved.

In this study, we used the Chinese herbal medicine Xianhuayin to treat buccal mucosal premalignant lesions in hamsters. Studies have shown that Chinese herbal medicines can improve the circulatory system and function of immune cells, which is helpful for the recovery of mucosal epithelium (Wu et al., 2008). The main component of glycyrrhiza is licorice zinc (Zheng and Zhai, 2006), which can facilitate the regeneration of epithelial 
cells on the gastrointestinal tract. We showed in this study that after intragastric administration with Chinese herbal medicine Xianhuayin, the mucosal ruffles in the cheek pouch were reduced, the surface of the mucosa became smooth, congestion and anabrotic changes disappeared. Light and electron microscopic analysis showed that the layer of the disorderly arranged epithelium became clear, basal layers of the cells were arranged regularly, the shape and border of the cells became distinct, honeycomb structure appeared in most of the cell surfaces and the widening intercellular gap was reduced. These results suggested that significant therapeutic efficacy was achieved for the hamsters in Xianhuayin-treated group compared to those in untreated premalignant lesion and NS-treated groups.

Based on the previous studies, we generated a premalignant lesion model in the oral mucosa of hamsters with the use of DMBA in acetone, producing lesions consistent with previous studies (Li et al., 2007).

By light and electron microscopic analysis for the morphological changes after treatment, we found that Xianhuayin played a significant role in the reversal of oral premalignant lesions. The underlying mechanisms may be related to its anti-inflammatory properties, increased immunity and facilitation of anabrotic healing.

\section{Conclusion}

We conclude that Xianhuayin is effective in the reversal of DMBA-induced premalignant lesions in the oral cheek pouch of golden hamsters. The underlying mechanisms of this effect must be investigated and which may include the antiinflammatory properties of Xianhuayin, increased immunity and facilitation of anabrotic healing. Which compoents of Xianhuayin are involved in the observed effects remain to be discovered. Chinese herbal medicine Xianhuayin may become a safe and effective drug for the treatment of oral premalignant lesions.

\section{Acknowledgements}

This work was supported by grants from the highly distinguished Oncology Subject Foundation of Colleges in Hebei Province.

\section{References}

The Ministry of Science and Technology of the People's Republic of China (2006). Guidance suggestion of caring laboratory animals. 2006-09-30.

Xie JF, Zhang KH (1989). Athymic mouse transplantation of experimental oral mucosa premalignant lesion. Chin J Stomatol, 24(3): 149-151.

Zhou CT, Zhang SL (1997). Pathological characteristic of 12 item epithelial dysplasia in golden hamster by light microscope analysis. Shanghai J Stomatol, 6(1): 32.

Xu YZ, Xin XH, Liu YX, Liu TL, Wang D (2009). An effect of Chinese herbal medicine Xianhuayin expression on activation regulatory factor of the patient with oral lichen planus. Shandong J Traditional Chin Med, 28(1): 13-14.

Xu YZ, Li SC, Wang XL, Li SL, Zhang KH, Zhang PH (2001). An experimental study of the effects of natural carotene on DMBA - induced oral buccal mucosa premalignant lesion in golden hamsters. J Mod Stomatol, 15(3): 174-175.

Buajeeb W, Poomsawat S, Punyasingh J, Sanguansin S (2009). Expression of P16 in oral cancer and premalignant lesions. Oral Pathol Med, 38(1): 104-108.

Gao Y (2008). New classification and advancement of oral mucosa premalignant lesion histology of WHO. Chin J Pract Stomatol, 1(12): 705-708.

Wu L, Zhou CT, Wu F, Li MY (2008). Clinical and microbiological study of compound of light yellow Sophora root collutory on treatment of oral lichen planus. Shanghai J Stomatol, 17(2): 118-120.

Zheng LG, Zhai SD (2006). A study of therapeutical effect and mucous membrane irritation of licorzinc film on experimental dental ulcer. Chin J New Drugs, 15(8): 601-603.

Li F, Sun Z, Wu HR, Chen XX, Li N (2007). Chemoprevention of Zyflamend on experimental lesion precancerous of oral mucosa. Beijing J Stomatol, 15(2): $61-64$.

\footnotetext{
*Corresponding authors: Yan-zhi Xu

Address: Department of Stomatology, The Fourth Hospital of Hebei Medical University, Shijiazhuang 050011, China

Tel: 86031186095436 E-mail: xu_yanzhi@163.com
} 